\title{
Holographic entanglement entropy in general holographic superconductor models
}

\author{
Yan Peng ${ }^{a}$ and Qiyuan $\operatorname{Pan}^{b}$ \\ ${ }^{a}$ School of Mathematics and Computer Science, Shaanxi University of Technology, \\ Hanzhong, Shaanxi 723000, P.R. China \\ ${ }^{b}$ Institute of Physics and Department of Physics, Hunan Normal University, \\ Changsha, Hunan 410081, P.R. China \\ E-mail: yanpengphy@163.com, panqiyuan@126.com
}

ABSTRACT: We study the entanglement entropy of general holographic dual models both in AdS soliton and AdS black hole backgrounds with full backreaction. We find that the entanglement entropy is a good probe to explore the properties of the holographic superconductors and provides richer physics in the phase transition. We obtain the effects of the scalar mass, model parameter and backreaction on the entropy, and argue that the jump of the entanglement entropy may be a quite general feature for the first order phase transition. In strong contrast to the insulator/superconductor system, we note that the backreaction coupled with the scalar mass can not be used to trigger the first order phase transition if the model parameter is below its bottom bound in the metal/superconductor system.

KeYwords: Gauge-gravity correspondence, Holography and condensed matter physics (AdS/CMT)

ARXIV EPRINT: 1404.1659 


\section{Contents}

1 Introduction 1

2 General superconductor in AdS soliton $\quad 2$

2.1 Bulk equations of motion and boundary conditions 2

2.2 Holographic entanglement entropy in insulator/superconductor transition 4

3 General superconductor in AdS black hole $\quad 7$

$\begin{array}{lll}3.1 & \text { Bulk equations of motion and boundary conditions } & 7\end{array}$

3.2 Holographic entanglement entropy in superconductor transition 8

$\begin{array}{lll}4 & \text { Conclusions } & 10\end{array}$

\section{Introduction}

The anti-de Sitter/conformal field theories (AdS/CFT) correspondence provides us a holographic dual description of the strongly coupled field theories with a weakly coupled gravitational system [1-3]. In recent years, this correspondence has been applied to study the holographic superconductor model, which is constructed by a scalar field coupled to a Maxwell field in an AdS black hole background [4-6]. It shows that the black hole becomes unstable and the scalar field condensates beyond the horizon below a critical temperature. According to AdS/CFT correspondence, the instability of the bulk black hole is dual to the conductor and superconductor phase transition. Recently, the holographic dual of the insulator and superconductor system is also established in the background of an AdS soliton [7]. Due to the potential applications to the condensed matter physics, these gravity duals attracted a lot of attention and many properties have been disclosed, see for examples [8]-[26].

The instability in the holographic superconductor models usually corresponds to the second order phase transition. It was stated in $[27,28]$ that the holographic superconductor via the Stückelberg mechanism allows the first order phase transition to occur when the model parameter surpasses some threshold value. Some interesting extensions were done in $[29,30]$ by including backreaction. It was found that the backreaction can trigger the first order phase transition when the Stückelberg model parameter is below its critical threshold. It was announced in [31] that the Stückelberg mechanism together with the backreaction will determine the order of phase transition when applying the Stückelberg mechanism to the AdS soliton spacetime. Generally speaking, there is only the second order phase transition for different masses of the scalar field in the probe limit [32]. Since the order of phase transition desponds on the choices of the couplings and the mass of the scalar field is crucial to the formation of the scalar hair in the superconductor model, it is 
interesting to explore the effect of the scalar mass $m$ on the order of phase transition in the Stückelberg model especially with backreaction.

On the other hand, the entanglement entropy is a powerful tool to keep track of the degrees of freedom in a strongly coupled system when other traditional probes might not be available. According to the AdS/CFT correspondence, the entanglement entropy may provide us new insights into the quantum structure of the spacetime [33, 34]. Ryu and Takayanagi $[35,36]$ have presented a proposal to compute the entanglement entropy of CFTs from the minimal area surface in the gravity side. This proposal provides a simple and elegant way to calculate the entanglement entropy of a strongly coupled system which has a gravity dual. Since then, there have been a lot of works studying the entanglement entropy in various gravity theories [37-45]. Extending the investigation to the holographic superconductors, Albash and Johnson observed in the metal/superconductor system that the entanglement entropy in superconducting case is always less than the one in the metal phase and the entropy as a function of temperature is found to have a discontinuous slop at the transition temperature $T_{c}$ in the case of the second order phase transition [46]. However, there is a discontinuous jump in the entropy when including the first order phase transition [46], which means that the entropy can be used to determine the order of phase transition. Cai et al. investigated the entanglement entropy of the holographic $p$-wave superconductor phase transition and observed the second order, first order and zeroth order phase transitions from the behavior of the entanglement entropy at some intermediate temperatures $[47,48]$. Arias and Landea computed the holographic entanglement entropy for both $p$ and $p+i p$ systems, and showed that the entanglement entropy satisfies an area law [49]. More recently, Kuang et al. examined the properties of the entanglement entropy in the four-dimensional AdS black hole and found that near the contact interface of the superconducting to normal phase the entanglement entropy has a different behavior due to the proximity effect [50]. In the insulator/superconductor transition, it is shown that the entanglement entropy for a half space first increases and reaches its maximum at a certain chemical potential and then decreases monotonically as chemical potential increases [51-54]. As a further step along this line, it is of great interest to generalize the investigation on the entanglement entropy of general holographic superconductors via the Stückelberg mechanism and study systematically the effects of the mass, model parameter and backreaction on the entropy. Furthermore we want to obtain some general feature for the entanglement entropy of the holographic dual models both in the backgrounds of the AdS soliton and AdS black hole.

The outline of this work is as follows. In section II, we will study the entanglement entropy of the general superconductors in the AdS soliton. In section III, we will extend our discussion to the AdS black hole. We will conclude our main results in the last section.

\section{General superconductor in AdS soliton}

\subsection{Bulk equations of motion and boundary conditions}

In the probe limit, it was argued that only second order phase transition can happen in the AdS soliton background [7]. Considering the backreaction of the matter field to the 
background, it was found that strong backreaction can bring about first order phase transition [25]. Applying the Stückelberg mechanism to the soliton configuration, it concluded that when the backreaction of the matter field becomes weaker, the Stückelberg parameter combined with the backreaction can accommodate the first order phase transition to occur [31]. Applying the Stückelberg mechanism to insulator/superconductor phase transition in the five-dimensional AdS soliton spacetime, it stated in [52] that the entanglement entropy serves as a good probe to the order of phase transition. We will generalize the discussion in $[51,52,54]$ to the more general superconductor by choosing various masses $m$ and charges $q$ of the scalar field, and examining the formation of scalar hair in another Stückelberg superconductor model which is different from that in ref. [52].

The generalized Stückelberg Lagrange density reads [31]

$$
\mathcal{L}=R+\frac{(d-1)(d-2)}{L^{2}}-\frac{1}{4} F^{\mu \nu} F_{\mu \nu}-(\partial \psi)^{2}-m^{2}|\psi|^{2}-F(\psi)(\partial p-q A)^{2},
$$

where $\psi(r)$ and $A_{\mu}$ are the scalar and Maxwell fields, $d$ is the dimensionality of the spacetime, and $L$ is the AdS radius which will be scaled unity in our calculation. Here we will change the strength of backreaction with the charge of the scalar field $q$. When $q \rightarrow \infty$ with the fixed $q \psi$ and $q \phi$, the backreaction of the matter fields becomes negligible and the metric solutions reduce to the pure AdS soliton spacetime. For the general function $F(\psi)$, in contrast to $F(\psi)=\psi^{2}+\zeta \psi^{6}$ discussed in ref. [52], we will choose $F(\psi)=\psi^{2}+q^{2} c_{4} \psi^{4}[31]$ in this work, where $c_{4}$ is the model parameter. Setting $q A=\hat{A}$ and considering the gauge symmetry $\hat{A} \rightarrow \hat{A}+\partial \Lambda$ and $p \rightarrow p+\Lambda$, we can fix the gauge $p=0$ by using the gauge freedom.

Since we are interested in including the backreaction, we will choose the metric in the form [25]

$$
d s^{2}=r^{2}\left[-e^{C(r)} d t^{2}+e^{D(r)} B(r) d \eta^{2}+d x^{2}+d y^{2}\right]+\frac{d r^{2}}{r^{2} B(r)},
$$

where we require that $B(r)$ vanishes at some radius $r_{0}$ which is the tip of the soliton. In order to smooth the solutions at the tip, we should impose a period $\kappa$ for the coordinate $\eta$

$$
\kappa=\frac{4 \pi e^{-D\left(r_{0}\right) / 2}}{r_{0}^{2} B^{\prime}\left(r_{0}\right)} .
$$

Choosing the Maxwell and scalar fields in the form

$$
A=\phi(r) d t, \quad \psi=\psi(r),
$$

we can obtain the equations of motion

$$
\begin{aligned}
\psi^{\prime \prime}+\left(\frac{5}{r}+\frac{B^{\prime}}{B}+\frac{C^{\prime}}{2}+\frac{D^{\prime}}{2}\right) \psi^{\prime}+\frac{q^{2} \phi^{2} e^{-C}}{r^{4} B}\left(\psi+2 q^{2} c_{4} \psi^{3}\right)-\frac{m^{2}}{r^{2} B} \psi & =0, \\
\phi^{\prime \prime}+\left(\frac{3}{r}+\frac{B^{\prime}}{B}-\frac{C^{\prime}}{2}+\frac{D^{\prime}}{2}\right) \phi^{\prime}-\frac{2 q^{2} \phi}{r^{2} B}\left(\psi^{2}+q^{2} c_{4} \psi^{4}\right) & =0, \\
C^{\prime \prime}+\frac{1}{2} C^{2}+\left(\frac{5}{r}+\frac{B^{\prime}}{B}+\frac{D^{\prime}}{2}\right) C^{\prime}-\left[\phi^{\prime 2}+\frac{2 q^{2} \phi^{2}}{r^{2} B}\left(\psi^{2}+q^{2} c_{4} \psi^{4}\right)\right] \frac{e^{-C}}{r^{2}} & =0,
\end{aligned}
$$




$$
\begin{gathered}
B^{\prime}\left(\frac{3}{r}-\frac{C^{\prime}}{2}\right)+B\left(\psi^{\prime 2}-\frac{1}{2} C^{\prime} D^{\prime}+\frac{e^{-C} \phi^{\prime 2}}{2 r^{2}}+\frac{12}{r^{2}}\right)+\frac{q^{2} \phi^{2} e^{-C}}{r^{4}}\left(\psi^{2}+q^{2} c_{4} \psi^{4}\right)+\frac{1}{r^{2}}\left(m^{2} \psi^{2}-12\right)=0 \\
D^{\prime}=\frac{2 r^{2} C^{\prime \prime}+r^{2} C^{\prime 2}+4 r C^{\prime}+4 r^{2} \psi^{\prime 2}-2 e^{-C} \phi^{\prime 2}}{r\left(6+r C^{\prime}\right)}
\end{gathered}
$$

Since the equations are coupled and nonlinear, we have to count on the numerical approach. We will integrate these equations from the tip $r_{0}$ out to the infinity.

At the tip, there are four independent parameters $r_{0}, \psi\left(r_{0}\right), \phi\left(r_{0}\right)$ and $C\left(r_{0}\right)$. Considering the two useful scaling symmetries

$$
\begin{aligned}
& r \rightarrow a r, \quad(t, \eta, x, y) \rightarrow(t, \eta, x, y) / a, \quad \phi \rightarrow a \phi, \\
& C \rightarrow C-2 \ln b, \quad t \rightarrow b t, \quad \phi \rightarrow \phi / b,
\end{aligned}
$$

we can adjust the solutions to satisfy $r_{0}=1$ and $C\left(r_{0}\right)=0$. At $r \rightarrow \infty$, after choosing $m^{2} \geqslant m_{B F}^{2}=-\frac{(d-1)^{2}}{4}=-4[55]$, the scalar and Maxwell fields have the form

$$
\psi=\frac{\psi_{-}}{r^{\lambda_{-}}}+\frac{\psi_{+}}{r^{\lambda_{+}}}+\cdots, \phi=\mu-\frac{\rho}{r^{2}}+\cdots,
$$

where $\lambda_{ \pm}=2 \pm \sqrt{4+m^{2}}$ are the conformal dimensions of the operators, $\mu$ and $\rho$ can be interpreted as the chemical potential and charge density in the dual theory respectively. We will fix $\psi_{-}=0$ and use $\psi_{+}=<O_{+}>$to describe the phase transition in the following discussion. In order to recover the pure AdS boundary, we also need $C(r \rightarrow \infty)=0$ and $D(r \rightarrow \infty)=0$. It should be noted that, after obtaining the solutions, we will scale them to satisfy $\kappa=\pi[25]$.

\subsection{Holographic entanglement entropy in insulator/superconductor transition}

In this section, we want to explore the properties of the phase transition through the topological entanglement entropy method. The authors in refs. $[35,36]$ have presented a proposal to compute the entanglement entropy of conformal field theories (CFTs) from the minimal area surface in gravity side. Consider a strongly coupled field theory with gravity dual, the entanglement entropy of subsystem $\bar{A}$ with its complement is given by searching for the minimal area surface $\gamma_{\bar{A}}$ in the bulk with the same boundary $\partial \bar{A}$ of a region $\bar{A}$. Then the entanglement entropy of $\bar{A}$ with its complement is given by

$$
S_{\bar{A}}=\frac{\operatorname{Area}\left(\gamma_{\bar{A}}\right)}{4 G_{N}}
$$

where $G_{N}$ is the Newton's constant in the bulk. For simplicity, we consider the entanglement entropy for a half space which corresponds to a subsystem $\bar{A}$ defined by $x>0$, $-\frac{R}{2}<y<\frac{R}{2}(R \rightarrow \infty), 0 \leq \eta \leq \kappa$. Then the entanglement entropy can be deduced from eq. $(2.13)$ as $[51,52,54]$

$$
S_{\bar{A}}^{h a l f}=\frac{R \kappa}{4 G_{N}} \int_{r_{0}}^{\frac{1}{\varepsilon}} r e^{\frac{D(r)}{2}} d r=\frac{R \pi}{8 G_{N}}\left(\frac{1}{\varepsilon^{2}}+S\right),
$$

where $r=\frac{1}{\varepsilon}$ is the UV cutoff. The first term is divergent as $\varepsilon \rightarrow 0$. In contrast, the second term does not depend on the cutoff and thus is physical important. As a matter of fact, 

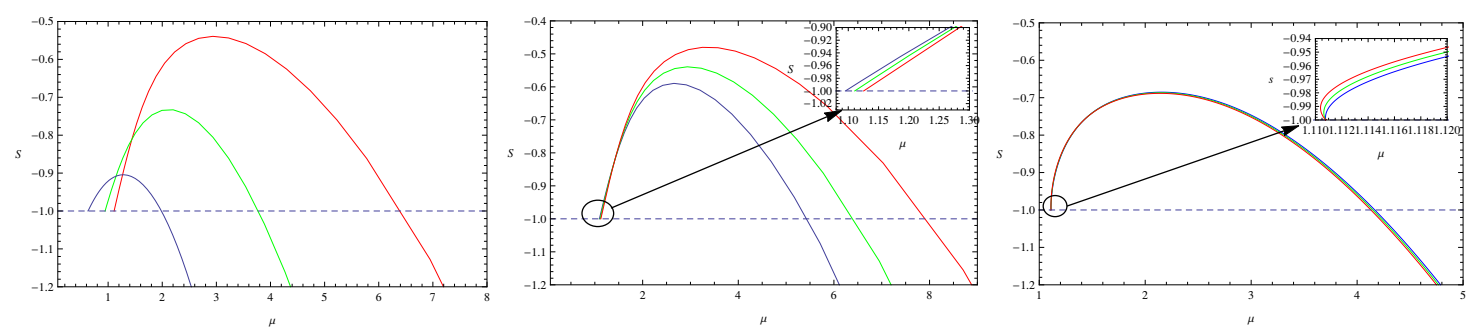

Figure 1. (Color online) The entanglement entropy as a function of the chemical potential $\mu$ for $\kappa=\pi$. The dashed blue line in each panel corresponds to the entropy without backreaction or the entropy of pure AdS soliton solution. The left panel is for the case $c_{4}=0, m^{2}=-15 / 4$ and the three lines from top to bottom correspond to increasing $q$, i.e., $q=1.7$ (red), $q=2$ (green) and $q=3$ (blue) respectively. The middle one shows the case $q=1.7, c_{4}=0$ and the three lines from top to bottom correspond to decreasing $m^{2}$, i.e., $m^{2}=-149 / 40$ (red), $m^{2}=-15 / 4$ (green) and $m^{2}=-151 / 40$ (blue) respectively. The right one presents the case $m^{2}=-15 / 4, q=1.7$, and the three lines correspond to decreasing $c_{4}$, i.e., $c_{4}=0.46$ (red), $c_{4}=0.45$ (green) and $c_{4}=0.44$ (blue) respectively.

this finite term is the difference between the entropy in the pure AdS soliton and the pure AdS space, and $S=-1$ corresponds to the pure AdS soliton.

Now we are in a position to study the effects of the charge $q$, mass $m$ and model parameter $c_{4}$ on the entanglement entropy. In the left panel of figure 1, we present the value of the entanglement entropy $S$ as a function of chemical potential $\mu$ with $c_{4}=0$, $m^{2}=-15 / 4$ for different charges $q$ in the superconductor phase. In order to compare with the result obtained in refs. [51, 52, 54], we also give the curve for the case $q=2$. From the picture, we can see that the entropy is a constant, i.e., $S=-1$ in the insulator phase. Note that there is a phase transition between the insulator and superconductor phases around the critical chemical potential $\mu_{c}$, i.e., $\mu_{c}$ refers to the onset of a phase transition. After condensate, i.e., $\mu>\mu_{c}$, the entropy first rises and arrives at its maximum as the chemical potential $\mu$ increases, then decreases monotonously. Obviously, for each value of the charge $q$, there is a discontinuity in the slope of $S$ at the critical chemical potential $\mu_{c}$, which indicates that the second order phase transition occurs. Furthermore, we find that as the charge $q$ decreases, the critical chemical potential $\mu_{c}$ and the maximum of the entropy $S$ become larger, which shows that the larger critical chemical potential $\mu_{c}$ corresponds to the larger maximum of the entropy $S$ after the scalar field condensates in the case of the second order phase transition.

In the middle panel of figure 1, we show the behavior of the entanglement entropy $S$ as a function of chemical potential $\mu$ with $q=1.7, c_{4}=0$ for different masses $m$. For each value of the mass $m$, after condensate, the entropy first rises and arrives at its maximum as the chemical potential $\mu$ increases, then decreases monotonously. Similar to the left panel, there is a discontinuity in the slope of $S$ at the critical chemical potential $\mu_{c}$, which can be regarded as the signature of the second order phase transition. Again, we see that the larger $\mu_{c}$ corresponds to the larger maximum of $S$ after condensate.

In the right panel of figure 1 , we plot the entanglement entropy $S$ as a function of 

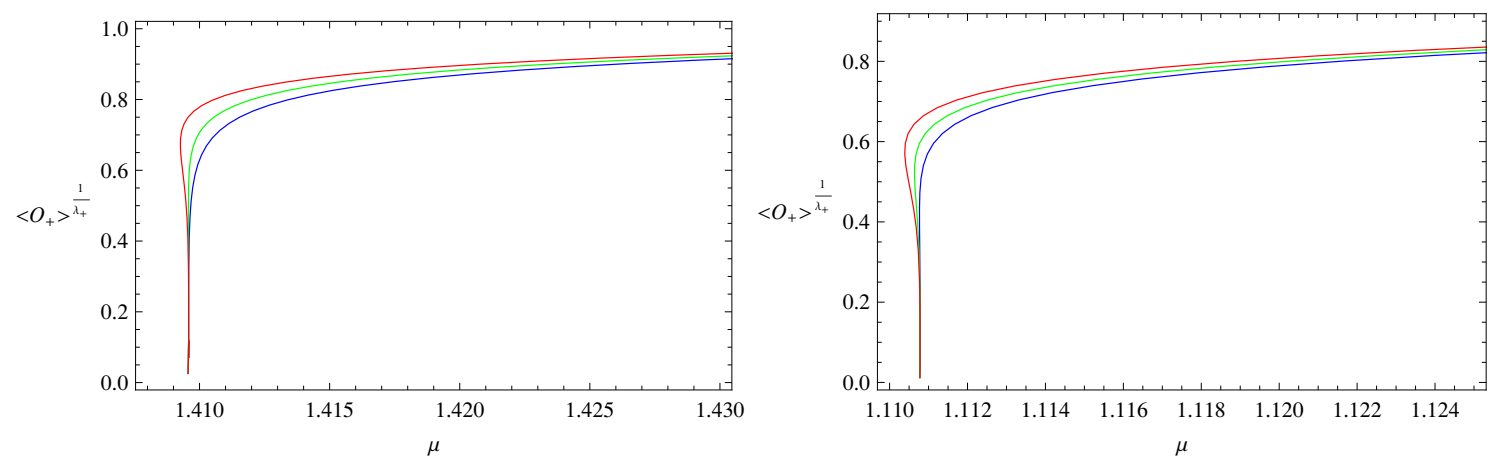

Figure 2. (Color online) The condensate $\left\langle O_{+}>^{\frac{1}{\lambda_{+}}}\right.$as a function of the chemical potential $\mu$ for $\kappa=\pi$. The three lines in the left panel from top to bottom correspond to decreasing $c_{4}$, i.e., $c_{4}=0.46$ (red), $c_{4}=0.45$ (green), $c_{4}=0.44$ (blue) for the fixed $m^{2}=-15 / 4$ and $q=1.7$. The three lines in the right one correspond to $c_{4}=0.26$ (red), $c_{4}=0.25$ (green), $c_{4}=0.24$ (blue) for the fixed $m^{2}=-3$ and $q=1.7$.

chemical potential $\mu$ with $m^{2}=-15 / 4, q=1.7$ for different model parameters $c_{4}$. We want to examine the entropy by allowing the first order phase transition to occur. Similar to the findings obtained in ref. [52], we find that the entropy becomes multivalued near the critical chemical potential $\mu_{c}$ when $c_{4} \geqslant 0.45$. Obviously, there is a sudden jump in the entropy, which indicates a first order phase transition there. This is in good agreement with the results in the left panel of figure 2, where we exhibit the condensate of $\left\langle O_{+}\right\rangle$for selected values of the charge $q$, mass $m$ and model parameter $c_{4}$. It should be noted that, when neglecting the backreaction of the matter fields on the background, the topological entropy is always a constant, i.e., $S=-1$ and we can not distinguish the order of phase transition.

For clarity, we also detect the effect of the mass $m$ on the condensation in this general insulator/superconductor model, which is missing in our previous work [31]. Choosing $q=1.7, m^{2}=-3$ and $-15 / 4$, we show the condensate $<O_{+}>^{1 / \lambda_{+}}$as a function of the chemical potential $\mu$ for different values of $c_{4}$ in figure 2 . We see that there is a threshold value $\bar{c}_{4}$ of $c_{4}$. When we enhance $c_{4}$ across the threshold, the condensate operator does not have a monotonic behavior, which indicates that the holographic insulator/superconductor system in AdS soliton experiences a first order phase transition. For the fixed $q=1.7$, we find $\bar{c}_{4}=0.25$ and $\bar{c}_{4}=0.45$ corresponds to the cases of $m^{2}=-3$ and $m^{2}=-15 / 4$ respectively, which means that the threshold value of $c_{4}$ will decrease as the mass $m^{2}$ increases. Thus, we conclude that for the fixed $q$ and $c_{4}$, the more negative mass $m^{2}$ make the first order phase transition harder to occur. Moreover, the appearance of the first order phase transition in figure 2 can be used to back up the numerical findings in the entanglement entropy $S$ shown in the right panel of figure 1. 


\section{General superconductor in AdS black hole}

\subsection{Bulk equations of motion and boundary conditions}

It was announced in [46] that the belt entanglement entropy experiences a jump when allowing the first order phase transition to occur in the four-dimensional AdS black hole background. In this section, we will extend the discussion by including the first order phase transition through Stückelberg mechanism. Taking backreaction of the spacetime into account, we take the ansatz for the metric of the four-dimensional AdS black hole

$$
d s^{2}=-g(r) e^{-\chi(r)} d t^{2}+\frac{d r^{2}}{g(r)}+r^{2}\left(d x^{2}+d y^{2}\right) .
$$

It requires that $g(r)$ vanishes at some radius $r_{+}$which corresponds to the horizon of the black hole. So the Hawking temperature reads

$$
T_{H}=\frac{g^{\prime}\left(r_{+}\right) e^{-\chi\left(r_{+}\right) / 2}}{4 \pi} .
$$

Assuming the matter fields in the forms

$$
A=\phi(r) d t, \quad \psi=\psi(r),
$$

We can obtain equations of motion

$$
\begin{aligned}
\chi^{\prime}+\left[r \psi^{\prime 2}+\frac{r}{g^{2}} e^{\chi} \phi^{2}\left(\psi^{2}+q^{2} c_{4} \psi^{4}\right)\right] & =0, \\
g^{\prime}-\left(\frac{3 r}{L^{2}}-\frac{g}{r}\right)+r g\left[\frac{1}{2} \psi^{\prime 2}+\frac{1}{4 g} e^{\chi} \phi^{\prime 2}+\frac{m^{2}}{2 g} \psi^{2}+\frac{1}{2 g^{2}} e^{\chi} \phi^{2}\left(\psi^{2}+q^{2} c_{4} \psi^{4}\right)\right] & =0, \\
\phi^{\prime \prime}+\left(\frac{2}{r}+\frac{\chi^{\prime}}{2}\right) \phi^{\prime}-\frac{2\left(\psi^{2}+q^{2} c_{4} \psi^{4}\right)}{g} \phi & =0, \\
\psi^{\prime \prime}+\left(\frac{2}{r}-\frac{\chi^{\prime}}{2}+\frac{g^{\prime}}{g}\right) \psi^{\prime}-\frac{m^{2}}{g} \psi+\frac{1}{g^{2}} e^{\chi} \phi^{2}\left(\psi+2 q^{2} c_{4} \psi^{3}\right) & =0 .
\end{aligned}
$$

Using the shooting method, we can solve these equations of motion numerically by integrating them from the horizon out to the infinity.

At the horizon, there are four independent parameters $r_{+}, \psi\left(r_{+}\right), \phi^{\prime}\left(r_{+}\right)$and $\chi\left(r_{+}\right)$. Considering the symmetry

$$
r \rightarrow a r, \quad(t, x, y) \rightarrow(t, x, y) / a, \quad \phi \rightarrow a \phi, \quad g \rightarrow a^{2} g,
$$

we can adjust the solutions to satisfy $r_{+}=1$. At the asymptotic AdS boundary $(r \rightarrow \infty)$, after choosing $m^{2}$ above the BF bound $m^{2} \geq m_{B F}^{2}=-(d-1)^{2} / 4=-9 / 4$ [55], the scalar and Maxwell fields behave like

$$
\psi=\frac{\psi_{-}}{r^{\lambda_{-}}}+\frac{\psi_{+}}{r^{\lambda_{+}}}+\cdots, \phi=\mu-\frac{\rho}{r}+\cdots
$$

with $\lambda_{ \pm}=\left(3 \pm \sqrt{9+4 m^{2}}\right) / 2$. Just as in the models of AdS soliton, we also take $\psi_{-}=0$ and the scalar condensation is described by the operator $\psi_{+}=<O_{+}>$. After obtaining 
the superconducting solutions, we will take the transformation $q \psi \rightarrow \frac{1}{\sqrt{2}} \psi, q \phi \rightarrow \phi, \frac{c_{4}}{2} \rightarrow$ $c_{4}$, and use $\gamma=\frac{1}{q^{2}}$ to describe the strength of backreaction [12, 29]. Note that this transformation does not change the topological entanglement entropy and the order of phase transitions. When $\gamma \rightarrow 0$, i.e., $q \rightarrow \infty$ with the fixed $q \psi$ and $q \phi$, it reduces to the standard holographic model in the absence of backreaction [27-29].

\subsection{Holographic entanglement entropy in superconductor transition}

It was found in metal/superconductor system that the entanglement entropy in superconducting case is always less than the one in the metal phase and the entropy as a function of temperature is found to have a discontinuous slop at the transition temperature $T_{c}$ in the case of second order phase transition $[46,50]$. In this section, we want to continue the discussion by examining the effects of the backreaction $\gamma$, mass $m$ and model parameter $c_{4}$ on the entropy.

Consider the subsystem $\tilde{A}$ with a straight strip geometry described by $-\frac{l}{2} \leqslant x \leqslant$ $\frac{l}{2}, 0 \leq y \leq \tilde{L}$, where $l$ is defined as the size of region $\tilde{A}$ and $\tilde{L}$ is a regulator which can be set to infinity. Minimizing the area of hypersurface $\gamma_{\tilde{A}}$ whose boundary is the same as the stripe $\tilde{A}$, the entanglement entropy for a belt geometry can be expressed as [46]

$$
S=\int_{\varepsilon}^{z_{*}} d z \frac{z_{*}^{2}}{z^{2}} \frac{1}{\sqrt{\left(z_{*}^{4}-z^{4}\right) z^{2} g(z)}}-\frac{1}{\varepsilon},
$$

with

$$
\frac{l}{2}=\int_{\varepsilon}^{z_{*}} d z \frac{z^{2}}{\sqrt{\left(z_{*}^{4}-z^{4}\right) z^{2} g(z)}},
$$

where $z_{*}$ satisfies the condition $\left.\frac{d z}{d x}\right|_{z_{*}}=0$ with $z=\frac{1}{r}$ and the UV cutoff $r=\frac{1}{\varepsilon}$ has been taken into consideration.

The entanglement entropy as a function of temperature $T$ with different values of the backreaction $\gamma$ and mass $m$ for fixed $l=1$ and $c_{4}=0$ is shown in figure 3. We find that, away from the probe limit, i.e., $\gamma \neq 0$, there is a discontinuity in the slope of $S$ at the critical temperature, which indicates the second order phase transition to occur. After condensate, the entropy decreases monotonously, which is in agreement with the conclusion obtained in $[46,50]$. From the picture, it also can be concluded that the critical temperature increases if we decrease $\gamma$ or $m^{2}$. Furthermore, we can get a relation, i.e., the higher critical temperature corresponds to the smaller entropy. When $\gamma=0$, the topological entropy and its slope are continuous around the critical temperature. Thus, we can not determine the order of phase transition in the probe limit, which is reasonable since we have neglected the backreaction of matter fields on the metric.

Now we want to exhibit the behavior of the entanglement entropy if the first order phase transition appears. Generally speaking, the order of the phase transition strongly depends on the choice of coupling. Thus, in figure 4 we plot the condensate $\left\langle O_{+}\right\rangle$as a function of the temperature $T$ with fixed $\rho=1$ and $\gamma=0.1$ for different model parameters $c_{4}$. We observe that, in this Stückelberg model, the high correction of the scalar field $\psi^{4}$ 

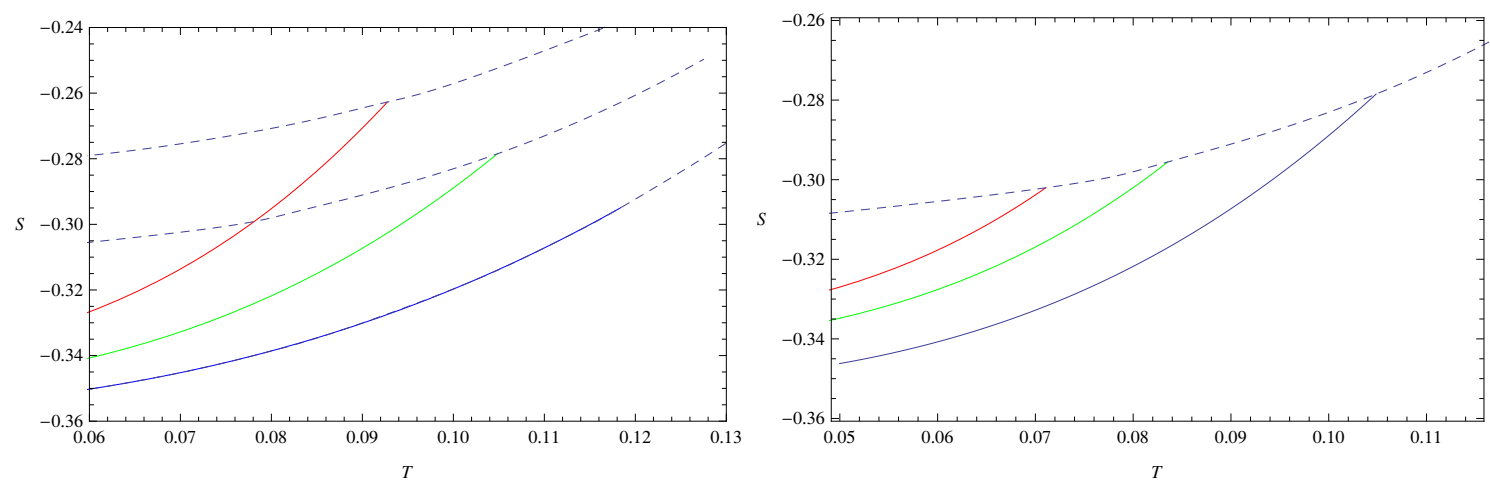

Figure 3. (Color online) The entanglement entropy as a function of temperature $T$ for fixed $l=1$ and $c_{4}=0$ with $\rho=1$. The left panel is for the case $m^{2}=-2$, the dashed line is from the ReissnerNordström AdS black holes and the solid curve is from the superconductor solutions. From top to bottom, the three sets of lines correspond to decreasing $\gamma$, i.e., $\gamma=0.2$ (red), $\gamma=0.1$ (green) and $\gamma=0$ (blue) respectively. Similarly, the right panel represents the case $\gamma=0.1$, and the three solid lines from top to bottom correspond to decreasing $m^{2}$, i.e., $m^{2}=-1$ (red), $m^{2}=-1.5$ (green) and $m^{2}=-2$ (blue) respectively.
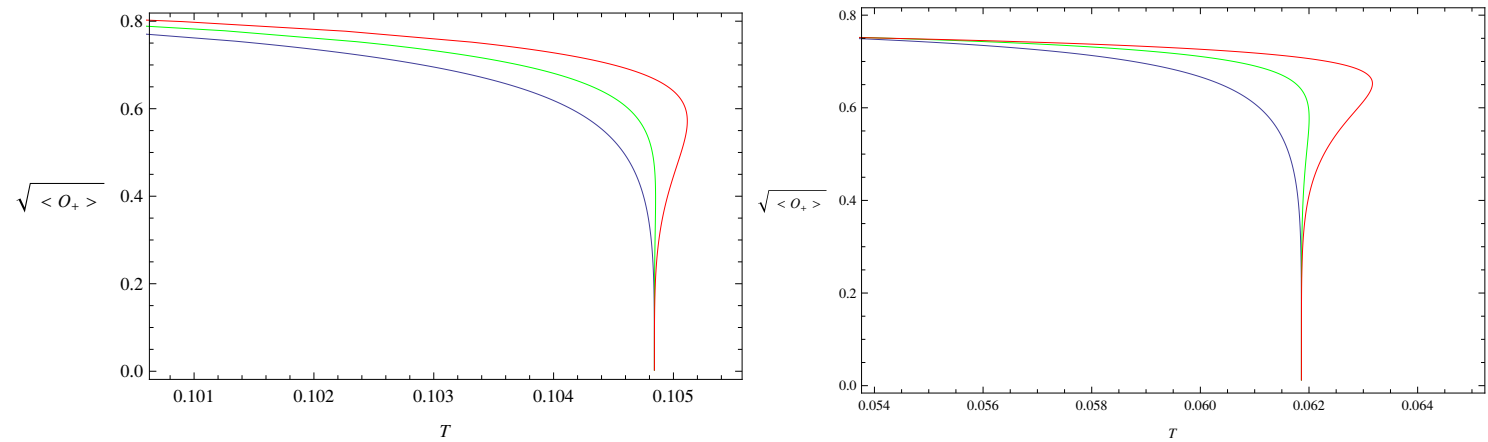

Figure 4. (Color online) The condensate $\left\langle O_{+}\right\rangle$as a function of the temperature $T$ for fixed $\gamma=0.1$ and $\rho=1$. The three lines in the left panel from right to left correspond to decreasing $c_{4}$ with the fixed $m^{2}=-2$, i.e., $c_{4}=0.8$ (red), $c_{4}=0.7$ (green), $c_{4}=0.6$ (blue), the right one is for $c_{4}=0.4$ (red), $c_{4}=0.3$ (green), $c_{4}=0.2$ (blue) with the fixed $m^{2}=-1 / 2$ respectively.

causes the first order phase transition for different values of $m^{2}$. We can easily obtain the threshold value $\bar{c}_{4}=0.7$ for the fixed $m^{2}=-2$ and $\bar{c}_{4}=0.3$ for the fixed $m^{2}=-1 / 2$. Above this threshold value, the condensate operator does not have a monotonic behavior, which indicates the appearance of first order phase transition. Correspondingly, we find that in figure 5 , where we show the entanglement entropy as a function of the temperature $T$ for fixed $\rho=1$ and $\gamma=0.1$, the entropy becomes multivalued near the critical temperature $T_{c}$ and there is a discontinuous jump in the entropy if $c_{4}>\bar{c}_{4}$. This means that, similar to the findings in [52], the entropy can distinguish the order of phase transition in our general superconductor model. It is interesting to note that the jump of the entanglement entropy may be a quite general feature for the first order phase transition.

From above discussion, we note that the entanglement entropy can be used to deter- 

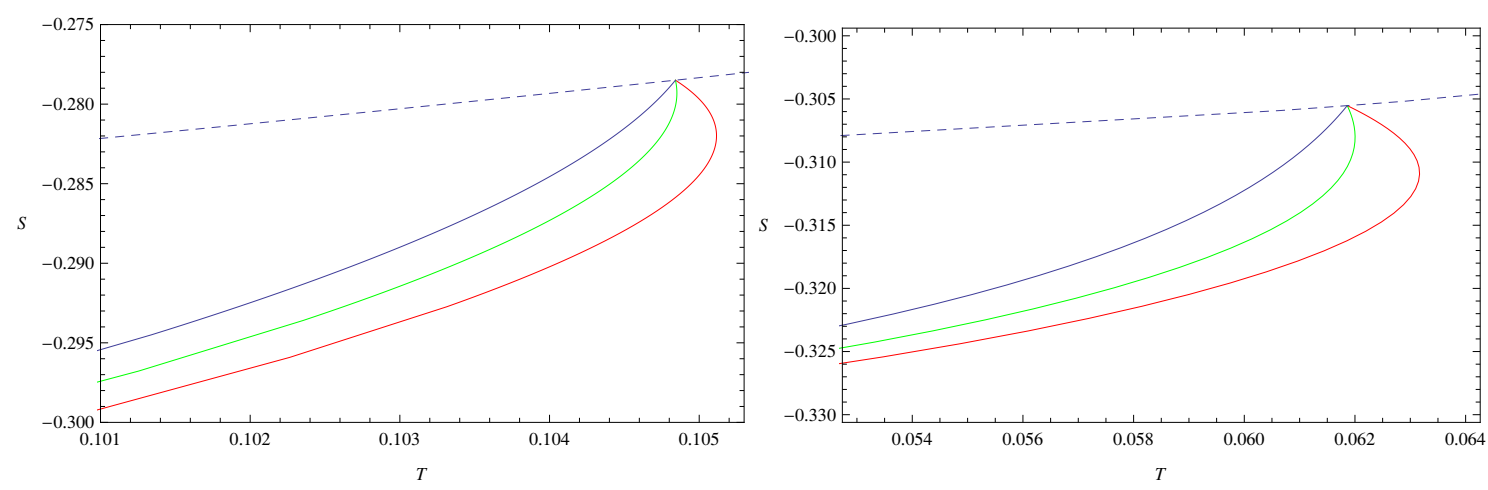

Figure 5. (Color online) The entanglement entropy as a function of the temperature $T$ for fixed $l=1$ and $\gamma=0.1$ with $\rho=1$. The dashed line is from the Reissner-Nordström AdS black holes and the solid curve is from the superconductor solutions. The three solid lines in the left panel from right to left correspond to decreasing $c_{4}$ with the fixed $m^{2}=-2$, i.e., $c_{4}=0.8$ (red), $c_{4}=0.7$ (green), $c_{4}=0.6$ (blue), the right one is for $c_{4}=0.4$ (red), $c_{4}=0.3$ (green), $c_{4}=0.2$ (blue) with the fixed $m^{2}=-1 / 2$ respectively.

mine the threshold value $\bar{c}_{4}$. Thus, in order to see the effects of the backreaction $\gamma$, mass $m$ on $\bar{c}_{4}$ more clearly, we plot $\bar{c}_{4}$ as a function of the scalar mass $m^{2}$ for different backreactions $\gamma$ in figure 6 by calculating the entanglement entropy of the system. It is found that for each fixed $\gamma, \bar{c}_{4}$ decreases as we increase $m^{2}$. That is to say that the more negative mass will depress the first order phase transition. On the other hand, for the chosen $m^{2}$, it is shown that $\bar{c}_{4}$ decreases as we increase $\gamma$ in the range [0,0.57], but it increases very slightly as we increase $\gamma$ when $\gamma>0.57$. Our more precise calculation shows that $\bar{c}_{4} \rightarrow 1.74$ when $\gamma=0$ and $m^{2} \rightarrow-9 / 4$, and $\bar{c}_{4}=0.05$ if $\gamma=0.57$ and $m^{2}=0$, which means that there are an upper limit of this threshold $\bar{c}_{4}=1.74$ and a bottom bound $\bar{c}_{4}=0.05$. Above this upper limit, there is only the first order phase transition for all choice of $m^{2}$ satisfying the BF bound. If $c_{4} \in[0.05,1.74]$, we observe that $c_{4}$ combined with $m^{2}$ and $\gamma$ can trigger the first order phase transition. Below the bottom bound, there is always the second order phase transition, which indicates that we can not rely on the backreaction coupled with the scalar mass to trigger the first order phase transition with $c_{4}<0.05$. This is totally different from the insulator/superconductor transition model in the AdS soliton where the strong backreaction can always trigger the first order phase transition [31]. Obviously, the entanglement entropy is a good probe to explore the property of the holographic dual models.

\section{Conclusions}

We have introduced a general class of gravity dual models via Stückelberg mechanism and investigated the behavior of the entanglement entropy of the systems both in the backgrounds of the AdS soliton and AdS black hole. We noted that the holographic entanglement entropy is a good probe to explore the properties of the phase transition. In the AdS soliton background, by calculating the holographic entanglement entropy for a half space in the insulator/superconductor transition, we found that the larger critical chemical 


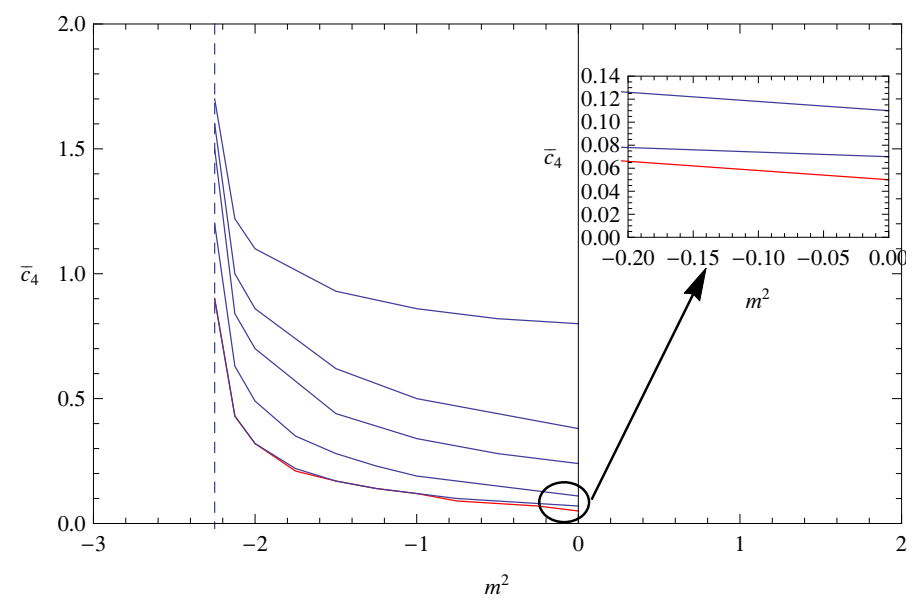

Figure 6. (Color online) The threshold value $\bar{c}_{4}$ as a function of the scalar mass $m^{2}$ for different backreactions $\gamma$. The lines from top to bottom correspond to the values of $\gamma$, i.e., $\gamma=$ $0,0.05,0.10,0.20,0.70$ and 0.57 (red). The vertical dashed line is for the case $m^{2}=m_{B F}^{2}=-9 / 4$. Note that the lines correspond to $\gamma=0.57$ and $\gamma=0.7$ almost coincide with each other.

potential corresponds to the larger maximum of the entropy after the scalar field condensates in the case of the second order phase transition. Furthermore, we observed that the backreaction coupled with the scalar mass and the model parameter can determine the order of phase transition and the more negative mass will make the first order phase transition harder to happen. Extending our calculation into the AdS black hole background, we obtained the effects of the backreaction, the scalar mass and the model parameter on the holographic entanglement entropy for a strip shape. If the model parameter $c_{4}$ larger than some threshold value determined by the backreaction and the scalar mass, we saw that the entropy becomes multivalued near the critical temperature and there is a discontinuous jump in the entropy, which indicates the appearance of first order phase transition. We argued that the jump of the entanglement entropy may be a quite general feature for the first order phase transition. It is also interesting to note that we can not rely on the backreaction coupled with the scalar mass to trigger the first order phase transition if the model parameter is below its bottom bound, which is totally different from the insulator/superconductor transition model in the AdS soliton where the strong backreaction can always trigger the first order phase transition.

\section{Acknowledgments}

We thank Professor Bin Wang for his helpful discussions and suggestions. This work was supported by the National Natural Science Foundation of China under Grant Nos. 11305097 and 11275066; the education department of Shaanxi province of China under Grant No. 2013JK0616; Hunan Provincial Natural Science Foundation of China under Grant No. 12JJ4007. 
Open Access. This article is distributed under the terms of the Creative Commons Attribution License (CC-BY 4.0), which permits any use, distribution and reproduction in any medium, provided the original author(s) and source are credited.

\section{References}

[1] J.M. Maldacena, The Large- $N$ limit of superconformal field theories and supergravity, Adv. Theor. Math. Phys. 2 (1998) 231 [Int. J. Theor. Phys. 38 (1999) 1113] [hep-th/9711200] [INSPIRE].

[2] S.S. Gubser, I.R. Klebanov and A.M. Polyakov, Gauge theory correlators from noncritical string theory, Phys. Lett. B 428 (1998) 105 [hep-th/9802109] [InSPIRE].

[3] E. Witten, Anti-de Sitter space and holography, Adv. Theor. Math. Phys. 2 (1998) 253 [hep-th/9802150] [INSPIRE].

[4] S.A. Hartnoll, Lectures on holographic methods for condensed matter physics, Class. Quant. Grav. 26 (2009) 224002 [arXiv:0903.3246] [INSPIRE].

[5] C.P. Herzog, Lectures on Holographic Superfluidity and Superconductivity, J. Phys. A 42 (2009) 343001 [arXiv:0904.1975] [InSPIRE].

[6] G.T. Horowitz, Introduction to Holographic Superconductors, Lect. Notes Phys. 828 (2011) 313 [arXiv:1002.1722] [INSPIRE].

[7] T. Nishioka, S. Ryu and T. Takayanagi, Holographic Superconductor/Insulator Transition at Zero Temperature, JHEP 03 (2010) 131 [arXiv:0911.0962] [INSPIRE].

[8] E. Nakano and W.-Y. Wen, Critical magnetic field in a holographic superconductor, Phys. Rev. D 78 (2008) 046004 [arXiv:0804.3180] [INSPIRE].

[9] G. Koutsoumbas, E. Papantonopoulos and G. Siopsis, Exact Gravity Dual of a Gapless Superconductor, JHEP 07 (2009) 026 [arXiv:0902.0733] [INSPIRE].

[10] J. Sonner, A Rotating Holographic Superconductor, Phys. Rev. D 80 (2009) 084031 [arXiv: 0903.0627] [INSPIRE].

[11] S.S. Gubser, C.P. Herzog, S.S. Pufu and T. Tesileanu, Superconductors from Superstrings, Phys. Rev. Lett. 103 (2009) 141601 [arXiv:0907.3510] [INSPIRE].

[12] S.A. Hartnoll, C.P. Herzog and G.T. Horowitz, Holographic Superconductors, JHEP 12 (2008) 015 [arXiv:0810.1563] [INSPIRE].

[13] Y. Liu, Q. Pan and B. Wang, Holographic superconductor developed in BTZ black hole background with backreactions, Phys. Lett. B 702 (2011) 94 [arXiv:1106.4353] [INSPIRE].

[14] Y. Peng, X.-M. Kuang, Y. Liu and B. Wang, Phase transition in the holographic model of superfluidity with backreactions, arXiv:1204.2853 [INSPIRE].

[15] J.P. Gauntlett, J. Sonner and T. Wiseman, Holographic superconductivity in M-theory, Phys. Rev. Lett. 103 (2009) 151601 [arXiv:0907.3796] [INSPIRE].

[16] J. Jing and S. Chen, Holographic superconductors in the Born-Infeld electrodynamics, Phys. Lett. B 686 (2010) 68 [arXiv:1001.4227] [INSPIRE].

[17] C.P. Herzog, An Analytic Holographic Superconductor, Phys. Rev. D 81 (2010) 126009 [arXiv: 1003.3278] [INSPIRE]. 
[18] K. Maeda, M. Natsuume and T. Okamura, Universality class of holographic superconductors, Phys. Rev. D 79 (2009) 126004 [arXiv:0904.1914] [InSPIRE].

[19] R. Gregory, S. Kanno and J. Soda, Holographic Superconductors with Higher Curvature Corrections, JHEP 10 (2009) 010 [arXiv:0907.3203] [INSPIRE].

[20] Q. Pan, B. Wang, E. Papantonopoulos, J. Oliveira and A.B. Pavan, Holographic Superconductors with various condensates in Einstein-Gauss-Bonnet gravity, Phys. Rev. D 81 (2010) 106007 [arXiv:0912.2475] [inSPIRE].

[21] X.-H. Ge, B. Wang, S.-F. Wu and G.-H. Yang, Analytical study on holographic superconductors in external magnetic field, JHEP 08 (2010) 108 [arXiv:1002.4901] [INSPIRE].

[22] Y. Brihaye and B. Hartmann, Holographic Superconductors in 3+1 dimensions away from the probe limit, Phys. Rev. D 81 (2010) 126008 [arXiv:1003.5130] [INSPIRE].

[23] S.S. Gubser, Breaking an Abelian gauge symmetry near a black hole horizon, Phys. Rev. D 78 (2008) 065034 [arXiv:0801.2977] [InSPIRE].

[24] S.A. Hartnoll, C.P. Herzog and G.T. Horowitz, Building a Holographic Superconductor, Phys. Rev. Lett. 101 (2008) 031601 [arXiv:0803.3295] [inSPIRE].

[25] G.T. Horowitz and B. Way, Complete Phase Diagrams for a Holographic Superconductor/Insulator System, JHEP 11 (2010) 011 [arXiv:1007.3714] [INSPIRE].

[26] N. Bobev, A. Kundu, K. Pilch and N.P. Warner, Minimal Holographic Superconductors from Maximal Supergravity, JHEP 03 (2012) 064 [arXiv:1110.3454] [INSPIRE].

[27] S. Franco, A. Garcia-Garcia and D. Rodriguez-Gomez, A General class of holographic superconductors, JHEP 04 (2010) 092 [arXiv:0906.1214] [INSPIRE].

[28] S. Franco, A.M. Garcia-Garcia and D. Rodriguez-Gomez, A Holographic approach to phase transitions, Phys. Rev. D 81 (2010) 041901 [arXiv:0911.1354] [INSPIRE].

[29] Q. Pan and B. Wang, General holographic superconductor models with Gauss-Bonnet corrections, Phys. Lett. B 693 (2010) 159 [arXiv: 1005.4743] [INSPIRE].

[30] Y. Peng, and Q.Y. Pan, Stc̈kelberg Holographic Superconductor Models with Backreactions, Commun. Theor. Phys. 59 (2013) 110.

[31] Y. Peng, Q. Pan and B. Wang, Various types of phase transitions in the AdS soliton background, Phys. Lett. B 699 (2011) 383 [arXiv:1104.2478] [INSPIRE].

[32] G.T. Horowitz and M.M. Roberts, Holographic Superconductors with Various Condensates, Phys. Rev. D 78 (2008) 126008 [arXiv:0810.1077] [INSPIRE].

[33] M. Van Raamsdonk, Comments on quantum gravity and entanglement, arXiv:0907.2939 [INSPIRE].

[34] M. Van Raamsdonk, Building up spacetime with quantum entanglement, Gen. Rel. Grav. 42 (2010) 2323 [arXiv: 1005.3035] [INSPIRE].

[35] S. Ryu and T. Takayanagi, Holographic derivation of entanglement entropy from AdS/CFT, Phys. Rev. Lett. 96 (2006) 181602 [hep-th/0603001] [INSPIRE].

[36] S. Ryu and T. Takayanagi, Aspects of Holographic Entanglement Entropy, JHEP 08 (2006) 045 [hep-th/0605073] [INSPIRE]. 
[37] T. Nishioka and T. Takayanagi, AdS Bubbles, Entropy and Closed String Tachyons, JHEP 01 (2007) 090 [hep-th/0611035] [INSPIRE].

[38] I.R. Klebanov, D. Kutasov and A. Murugan, Entanglement as a probe of confinement, Nucl. Phys. B 796 (2008) 274 [arXiv:0709.2140] [INSPIRE].

[39] A. Pakman and A. Parnachev, Topological Entanglement Entropy and Holography, JHEP 07 (2008) 097 [arXiv: 0805.1891] [INSPIRE].

[40] T. Nishioka, S. Ryu and T. Takayanagi, Holographic Entanglement Entropy: An Overview, J. Phys. A 42 (2009) 504008 [arXiv: 0905. 0932] [InSPIRE].

[41] L.-Y. Hung, R.C. Myers and M. Smolkin, On Holographic Entanglement Entropy and Higher Curvature Gravity, JHEP 04 (2011) 025 [arXiv:1101.5813] [INSPIRE].

[42] J. de Boer, M. Kulaxizi and A. Parnachev, Holographic Entanglement Entropy in Lovelock Gravities, JHEP 07 (2011) 109 [arXiv:1101.5781] [INSPIRE].

[43] N. Ogawa and T. Takayanagi, Higher Derivative Corrections to Holographic Entanglement Entropy for AdS Solitons, JHEP 10 (2011) 147 [arXiv:1107.4363] [INSPIRE].

[44] T. Albash and C.V. Johnson, Holographic Entanglement Entropy and Renormalization Group Flow, JHEP 02 (2012) 095 [arXiv: 1110.1074] [INSPIRE].

[45] R.C. Myers and A. Singh, Comments on Holographic Entanglement Entropy and RG Flows, JHEP 04 (2012) 122 [arXiv: 1202.2068] [INSPIRE].

[46] T. Albash and C.V. Johnson, Holographic Studies of Entanglement Entropy in Superconductors, JHEP 05 (2012) 079 [arXiv:1202.2605] [INSPIRE].

[47] R.-G. Cai, S. He, L. Li and Y.-L. Zhang, Holographic Entanglement Entropy on P-wave Superconductor Phase Transition, JHEP 07 (2012) 027 [arXiv: 1204.5962] [INSPIRE].

[48] L.-F. Li, R.-G. Cai, L. Li and C. Shen, Entanglement entropy in a holographic p-wave superconductor model, arXiv:1310.6239 [INSPIRE].

[49] R.E. Arias and I.S. Landea, Backreacting p-wave Superconductors, JHEP 01 (2013) 157 [arXiv: 1210.6823] [INSPIRE].

[50] X.-M. Kuang, E. Papantonopoulos and B. Wang, Entanglement Entropy as a Probe of the Proximity Effect in Holographic Superconductors, arXiv:1401.5720 [INSPIRE].

[51] R.-G. Cai, S. He, L. Li and Y.-L. Zhang, Holographic Entanglement Entropy in Insulator/Superconductor Transition, JHEP 07 (2012) 088 [arXiv:1203.6620] [INSPIRE].

[52] R.-G. Cai, S. He, L. Li and L.-F. Li, Entanglement Entropy and Wilson Loop in Stúckelberg Holographic Insulator/Superconductor Model, JHEP 10 (2012) 107 [arXiv:1209.1019] [INSPIRE].

[53] R.-G. Cai, L. Li, L.-F. Li and R.-K. Su, Entanglement Entropy in Holographic P-Wave Superconductor/Insulator Model, JHEP 06 (2013) 063 [arXiv:1303.4828] [INSPIRE].

[54] W. Yao and J. Jing, Holographic entanglement entropy in insulator/superconductor transition with Born-Infeld electrodynamics, JHEP 05 (2014) 058 [arXiv:1401.6505] [INSPIRE].

[55] P. Breitenlohner and D.Z. Freedman, Positive Energy in anti-de Sitter Backgrounds and Gauged Extended Supergravity, Phys. Lett. B 115 (1982) 197 [InSPIRE]. 